\title{
Un alegato en pro de una epistemología de las pruebas empíricas: ensayos clínicos aleatorios y posverdad
}

\author{
JUAN BAUTISTA BENGOETXEA* \\ Universidad de las Islas Baleares (España) \\ juanbautista.bengoechea@uib.cat
}

\begin{abstract}
Resumen
En este artículo propongo un análisis crítico de varias tendencias filosóficas contrarias al uso de la noción de verdad en el ámbito epistemológico, especialmente la de Rorty. En particular, sostengo que la verdad es un concepto clave para el estudio del conocimiento, especialmente el científico, en cuanto toma una forma concisa en los procedimientos experimentales de carácter probatorio-empírico. Gracias a este enfoque, busco socavar varias tesis de los enfoques de la 'posverdad' por medio del uso de un conjunto de procedimientos científicos de obtención de evidencias (ensayos clínicos aleatorios) en varios campos vinculados a la disciplina práctica de la medicina.
\end{abstract}

Palabras clave: posverdad; prueba empírica; RCT; medicina; epistemología; Rorty.

\section{A plea for an epistemology of evidence: randomized clinical trials and post-truth}

\begin{abstract}
In this paper, I present a critical analysis of several philosophical tendencies that go against the use of the notion of truth in epistemology, especially Rorty's one. Particularly, I claim that truth is a key concept for the study of knowledge, especially the scientific knowledge, as it takes a concise form through the experimental procedures for eliciting evidences. With the belp of this analysis, I seek to undermine some 'post-truth' theses by means of analyzing some scientific procedures to get evidences (randomized clinical trials) in several fields related to the practical discipline of medicine.
\end{abstract}

Key words: post-truth; evidence; RCT; medicine; epistemology; Rorty.

* Doctor en Filosofía y Profesor Titular del Departamento de Filosofía de la Universidad de las Islas Baleares. Algunas publicaciones del autor son Ética e ingeniería (2010, con C. Mitcham), 'Culture and Technology in Spain: From Philosophical Analysis to STS' (2006, con C. Mitcham) e 'Intuition and Evidential Facts in Carnap's Analysis of Space' (2019).

El presente texto de investigación es deudor del apoyo financiero de los Fondos FEDER para el Desarrollo Regional de la Comunidad Europea y del Ministerio de Ciencia, Innovación y Universidades (Gobierno de España, Agencia Estatal de Investigación (AEI)), así como del Proyecto de Investigación 'Estándares de prueba y elecciones metodólogicas en la fundamentación científica de las declaraciones de salud' (FFI2017-83543-P). El autor agradece el respaldo de todas las instituciones mencionadas. 


\section{INTRODUCCIÓN}

El discurso proceloso de mucho enfant terrible del siglo XXI nos dice una y otra vez que la verdad ya no tiene lugar, que es un artificio filosófico sin sustancia, un mero juego de palabras, que solo nos resta ir más allá hacia algo como la 'posverdad', sea lo que esto sea ${ }^{1}$. La decadencia de la filosofía es tan profunda, nos dicen, que o bien la rehacemos de arriba abajo, o bien le hacemos soltar el lastre más pesado y exasperante que la pudre. Y aunque resulte extraño para ciertos ojos, ese lastre pesado, contaminante y malicioso es, afirman, la propia filosofía o un tipo de esta (Anderson, 1996). Es esto lo que los 'nuevos compradores' de filosofía occidental de finales del milenio pasado nos pedían: reflexión nofilosófica - cuando no anti-filosófica-, aunque parezca inconcebible ${ }^{2}$.

Ante la consabida pérdida de peso intelectual que esto comportaría, nos ofrecen algo a cambio con tal de amortizar este gasto en prestigio cultural. Se trata de una forma distinta de reflexión, no novedosa, aunque sí re-situada. Nos piden que miremos a la filosofía con ojos reconvertidos, a modo de género de la literatura y nada más. Es más, recomiendan que la filosofía pierda su nombre y el de sus miembros más eminentes: la metafísica y la epistemología. Más le vale que se incorpore al mundo de la narrativa (Fuller, 2018: 20-23), que cuente relatos, cuentos, y abandone el uso de conceptos fantasmagóricos tales como la verdad, el progreso, el conocimiento cierto, la objetividad, la representación y las dicotomías afines que estos conllevan.

Sin embargo, sucede que no por etiquetar de 'literatura' a la filosofía esta se vaya a convertir en literatura. Tanto la literatura como la filosofía difícilmente son el resultado de una elección arbitraria y caprichosa de nomenclaturas más o menos ocurrentes. La literatura sería narrativa y poesía y ficción en general; relatos de historias y acontecimientos que suceden en algún lugar y tiempo. La filosofía, en cambio, se aproximaría más al análisis, al examen respaldado y cultivado por la racionalidad y las conceptualizaciones, a las hipótesis, en definitiva. Sería asimismo búsqueda de conocimiento, de objetividad según algunos, de la verdad según

1 Por supuesto, afirmar que la filosofía está enferma no es una idea nueva en absoluto. Acerca de la relación de varios autores 'posmodernos' (Heidegger, Lyotard, Foucault o Derrida) con esta afirmación, véase McIntyre (2018: 123-127).

2 Un referente ilustrativo de dicho 'relativismo' filosófico actual es Margolis (1991), pero no me detengo en su obra porque básicamente entiende que la posverdad equivale a la suspensión de la creencia ante el valor de verdad aplicado a los enunciados o al discurso. Esto no es nada nuevo en cuanto en lógica y en epistemología siempre se ha hablado de 'suspensión del juicio' cuando no podemos aplicarle el valor 'verdadero' o 'falso'. Decir que esto hace irrelevante la verdad es simplemente una exageración. 
otros. En suma, la filosofía persigue ser una disciplina de conocimiento, abstracta en su mayor parte, pero cuyos jueces últimos son la prueba empírica (evidencias) y la justificación y la argumentación (Haack, 2007: Cap. 8). ¿Cómo han llegado los críticos con la filosofía hasta el desideratum radical que obliga a elegir entre 'literatura o fenecimiento', si la divisoria entre filosofía y literatura siempre ha sido reconocida sin demasiados ambages?

A continuación, analizo críticamente la tendencia filosófica-literaria de Rorty que niega validez, incluso realidad, a la noción de verdad en el ámbito epistemológico (Sec. 1). Sostengo que la verdad es un concepto fundamental vinculado con el concepto de conocimiento, especialmente el científico, concepto que toma una forma concisa por medio de un tipo de procedimientos experimentales de carácter probatorio-empírico que sirven para socavar varias tesis de los enfoques de la 'posverdad', en particular de lo que denomino 'filosofía post-veritativa' (Sec. 2). Este tipo de procedimientos científicos de obtención de evidencias se denomina RCT (ensayos clínicos aleatorios) y se emplea habitualmente en ámbitos como la medicina, la farmacología, la alimentación o, en las ciencias humanas, la lingüística (Sec. 3). La coda final es una respuesta a las tesis previas 'negacionistas' de la verdad y una defensa de las epistemologías de las pruebas empíricas.

\section{CONTRA VERITAS: LA FILOSOFÍA LITERARIA DE RORTY}

Recordaba Pardo (2002: 11) que en cierta época hubo quienes comenzaron a hablar de la 'izquierda' y la 'derecha' filosóficas. La elección terminológica no tuvo demasiado éxito, pues siempre ha existido un continuum relativo, pero nos puede servir de orientación aquí. La derecha filosófica del siglo XX podría estar bien representada por la figura de Rudolf Carnap. Este empirista lógico y su credo filosófico sostuvo que la filosofía se debía someter a los hechos, tal y como es habitual en las ciencias naturales. Esto es, la naturaleza de la filosofía tenía que equivaler a la de una filosofía científica, al menos en su sentido de análisis lógico del lenguaje de la ciencia. En su primer texto mayor, Der logische Aurfbau der Welt (La construcción lógica del mundo) (1928), decía que

el autor no es dueño de la actitud y de la trayectoria reflexiva básicas de este libro, pues son parte del ambiente científico, algo que un individuo no puede generar ni asimilar por sí mismo. Las reflexiones aquí expuestas se alimentan del trabajo de ayudantes esforzados y receptivos. Lo que estos ayudantes comparten es una actitud científica básica. (Carnap, 1928: vi) 
Por su parte, la izquierda filosófica estaba compuesta por los nuevos dialécticos, próximos a la Escuela de Francfort, y entre otras cosas pensaban que los hechos que los empiristas lógicos tanto admiraban tenían que ser puestos en duda, si no eliminados del discurso del saber. Según ellos, la filosofía tenía que ser una crítica de dichos hechos. Consideraban que aceptar la existencia de 'hechos brutos' no era sino una forma vergonzante de legitimar el establishment sociocultural; esto es, de abonar acríticamente lo existente. No pensaban únicamente en la legitimación de eso que hay o existe y que sirve de tribunal del conocimiento, sino también y ante todo de un establishment político, social y cultural. Por ende, lo importante para la izquierda filosófica era tratar de modificar lo que no se podía cambiar según los parámetros de la derecha filosófica. Y precisamente fue en la filosofía científica de tintes analíticos donde identificaron los nuevos dialécticos la reserva más 'retrógrada' del conservadurismo filosófico. Al albur de las ideas de Walter Benjamin, se alejaron de todo lo que tuviese que ver con hechos, de lo fáctico. Esto les condujo al rechazo visceral y al alejamiento buscado respecto de todo filósofo empirista lógico, hasta el punto de insultar a esta escuela ${ }^{3}$.

Considerada así la cuestión, el desideratum de la aproximación de la filosofía a la literatura es marca de la izquierda filosófica. Parece que a la izquierda filosófica le interesa renegar de la ciencia, sobre todo de las ciencias naturales, a la hora de dar razón de la vida y del mundo. Podrían aceptar, a lo sumo, las ciencias sociales y humanas, vistas en cierto sentido como sucedáneo aceptable de la literatura. De ahí que Adorno y Horkheimer atendieran al psicoanálisis, o que Schopenhauer, Kierkegaard o Nietzsche avanzaran un pensamiento con estructura y forma literarias, incluso filológicas. Por el mismo motivo, a su vez, muchos filósofos parisinos comenzaron a desarrollar un existencialismo con tonos heideggerianos, hasta llegar a finales del siglo XX e inicios del XXI, cuando la etnometodología, la sociología de la ciencia y los estudios STS en general han llegado a optar por posiciones posverdad en las que la narrativa, los relatos y las ficciones son elementos clave.

Esta orientación se observa claramente a día de hoy entre los postestructuralistas de diverso cuño, desde los post-nietzscehanos y los post-

3 Fue en 'Der Neueste Angriff auf die Metaphysik' (1937) ('El ataque más novedoso contra la metafísica'), donde Max Horkheimer publicó los exabruptos más desquiciados contra Carnap y contra el Círculo de Viena. Fue en dichas páginas donde equiparó la tarea clarificadora conceptual de la filosofía científica con la limpieza étnica de los nazis -para vergüenza, quiero suponer, de los 'francfortianos' posteriores- (véase Horkheimer, 1937: 98, 101, 109, 122, 132). 
modernos hasta los neo-pragmatistas o los post-veritistas ${ }^{4}$. Es el desprecio a la filosofía que procura arreglárselas según estándares próximos a los de la ciencia natural y el aprecio ciego a una filosofía desconstruccionista (Derrida, 1987). Proyectaban el método desconstruccionista —de hecho, aceptable en la interpretación de textos literarios- a los escritos filosófico-científicos y a las actividades de laboratorio. Estos textos beligerantes que pretendidamente se aproximaban a una visión artística y literaria de la realidad favorecían una filosofía especulativa en la que las evidencias perdían peso.

Observemos no obstante algo de filosofía más sólida, con el fin de no parecer que nos concentramos únicamente en la caricatura, y veamos qué le piden a esta los filósofos. Un paladín entre estos ha sido Richard Rorty. Según este (Rorty, 2000: 55), el gremio filosófico norteamericano se dividió en dos bandos, el de los departamentos de ciencias (sociales, en su mayoría) y el de los de humanidades. Muchos filósofos no analíticos o continentales se dedicaron a criticar las ansias cientificistas de la filosofía y a desdeñar las ideas de las filosofías interesadas en las evidencias. Algunas de estas iniciativas tenían sentido, no así muchas otras. Un antecesor que influyó mucho en Rorty fue Martin Heidegger, quien en 1976 intentó que el bomo ciberneticus sustituyese a nuestra inveterada disciplina. Dicho en términos actuales, según Heidegger, lo que sustituiría a la filosofía sería la disciplina técnica desarrollada y empleada para planificar y organizar nuestra vida y trabajo. La ciencia y la técnica serían, por ende, el último paso o fase de la, hasta el momento, denominada 'filosofía'. Es decir, sería el límite final de la tradición occidental iniciada con Platón y finalizada con Nietzsche, el fin del discurso filosófico erigido sobre las representaciones lingüísticas de algo 'exterior' a nuestra mente (Nietzsche, 1873; Heidegger, 1976). La resolución heideggeriana, por lo tanto, proporcionaba dos trayectorias: una, desventurada pero muy real, era la de la ciencia y la tecnología; la otra, la que supondría la salvación del humano, era la del pensamiento nuevo. Según este, el ser humano auténtico debe pensar de una manera realmente reflexiva, buscando de forma profunda al ser del humano. Sería una forma de pensar alejada por completo, opuesta en realidad, a las formas de pensamiento platónico y próxima a la ruptura con licencias poéticas del lenguaje y sus reglas (véase Kusch, 1989: 193-201).

4 Tras la negación de la validez de la noción de verdad por los enfoques posverdad, surgió una tendencia filosófica conocida como 'veritismo', ante la cual hoy emergen los post-veritismos. Todo ello está tomando una forma espiral que parece dar razón a los mecanismos retóricos (no probatorios) de dominio del discurso filosófico y que no hacen gran favor a las filosofías que buscan, como es nuestro caso, evitar el juego del bullshit (véase Bengoetxea, 2017; Block, 2019: 30-34; Ball, 2017: Caps. 1-2). 
Hay quien podría pensar que Heidegger perseguía la renovación de la filosofía, no su eliminación. No lo creo, al igual que no creo que sea este el caso de las intenciones de Rorty. Si nos remitimos a sus escritos y conferencias, Rorty nos proporciona un 'logos' situado más allá de la filosofía - entrecomillo el término porque Rorty nunca lo aceptaría en su sentido clásico- . Rorty da por muerta la tradición platónica. Sin embargo, dado que afirmar que matamos la filosofía es algo demasiado crudo, prefiere hablarnos de la muerte de un tipo de filosofía (Rorty, 1998: 317). Pues bien, llamaré a esta filosofía la 'filosofía veritativa', la iniciada con Platón y desarrollada por Agustín, Descartes o Kant, entre otros. La filosofía que no es 'veritativa' es la filosofía literaria - la que solo tiene tradición (véase Moya, 2002: 317) - que denominaré 'filosofía post-veritativa'.

¿Qué tiene de filosofía la 'filosofía post-veritativa' de Rorty? Es un truismo decir que la filosofía se tiene que acercar al arte y a la literatura. Sería absurdo negarlo. No obstante, negar las funciones cognoscitivas de la filosofía, de sus indagaciones, de sus investigaciones, para vestirla meramente con ropajes literarios sería un ejercicio estético sin mucho sentido. Pero esta parece ser la opción rortyana cuando nos dice que el filósofo o bien se dedica a la ciencia - y la filosofía procura naturalizarse y, en gran medida, se hace ciencia- o bien se dedica a contar relatos, con lo que la 'filosofía post-veritativa' es en realidad literatura. El resto de la 'filosofía veritativa' - la epistemología y la ontología sobre todo- está de sobra.

\section{FILOSOFÍA POST-VERITATIVA Y POSVERDAD}

Entre 1979 y la primera década del siglo XXI, Rorty ha sido el adalid 'filosófico' de la 'cultura sin filosofía'. Obviamente, esta filosofía es la 'filosofía veritativa' que se asocia especialmente, al menos directamente, con Descartes y con Kant, verdaderos ejes del apuntalamiento de la filosofía como 'arquitectónica' de la cultura occidental. En esta tradición, Rorty observa que la filosofía europea era el faro cultural, el guardián social y el juez político. La filosofía era una disciplina cognoscitiva, un campo que procuraba aportar conocimientos empírico-abstractos y abstracto-formales; era una disciplina profesional en gran medida, sistemática y fundamentada en la epistemología. Esto es, la filosofía decía qué era conocimiento y qué no lo era; era la disciplina que juzgaba lo que la construcción de representaciones podía afirmar acerca del mundo, la que decidía qué era verdadero y qué no. En definitiva, era un constructo

\footnotetext{
5 Los artículos recopilados en Consequences of Pragmatism (1982) reflejan esto mismo, así como las explicaciones de Rorty sobre cómo concibe su actitud pragmatista.
} 
enorme, el fundamento de todo el conocimiento, ante todo del conocimiento científico, aquel que vigilaba rigurosamente la forma europea de entender la vida.

Pero la fábula terminó, dice Rorty, y es el momento de abandonarla completamente. La 'filosofía post-veritativa' debe ocupar su puesto y convertir el 'gran relato' en relatos pequeños, desperdigados y edificantes en torno a los cuales el conocimiento no será sino búsqueda de consensos (véase Lyotard, 1987). El pensador ahora será crítico literario: comparará relatos, generará conversaciones y participará en ellas, inventará nuevos diccionarios, léxicos, para ir clarificando los diversos tipos de problemas que acosan a la sociedad. En su nuevo rol, el filósofo no deberá aceptar algo como 'el último vocabulario'; la puesta a punto del léxico y su constante revisión pragmatista serán parte de un proceso sin fin. No hay un relato o tipo de relato que nos diga qué es lo real como tal, no hay un relato último. Todo relato y sus vocabularios serán contingentes, válidos en cierto periodo de tiempo, no eternamente. Cuando no precisen cuáles son los problemas del ser humano, de la gente, y cuando no ofrezcan soluciones más o menos plausibles, serán abandonados por resultar envejecidos, piensa Rorty. Este sería el caso de los relatos cuyas metáforas ya no nos sirven en nuestra vida, el caso de relatos fosilizados ante los cuales corremos el riesgo de pensar que son 'grandes relatos' que contienen verdades universales. Y precisamente es esto lo que la 'filosofía post-veritativa' debe diagnosticar y evitar a toda costa.

La 'filosofía veritativa', la que busca la veritas y el saber, ya no es la guía de nuestra vida psíquica y comportamental, no nos dirige. La razón se volvió sospechosa tras Nietzsche y la noción de poder se convirtió en eje de la reflexión de la sospecha ${ }^{6}$. Los pensadores literarios de la 'filosofía post-veritativa' no creen, sin embargo, que la 'filosofía veritativa' se librara de cierto impulso condescendiente ante sus lectores. También era, dicen, una filosofía consoladora, si bien sus remedios resultaron contaminantes. Nuestros defensores de la posverdad siguen creyendo que las pastillas de teoría platónica de las Ideas, de ontoteología cristiana, de ontoepistemología cartesiana o de elucidación conceptual del Círculo de Viena, entre otros tipos, tan solo han contaminado la filosofía hasta conducirla a su último suspiro. Los grandes pensadores de manual, considera Rorty, se han situado por encima de los demás mortales a modo de se-

$6 \quad$ En 1873 (Sobre verdad y mentira en sentido extramoral), Nietzsche ya sospechaba de la verdad y la trataba como "una hueste en movimiento de metáforas, metonimias, antropomorfismos; en resumidas cuentas, una suma de relaciones humanas que han sido realzadas, extrapoladas y adornadas poética y retóricamente y que, después de un prolongado uso, un pueblo considera firmes, canónicas y vinculantes" (véase Nietzsche, 1873 [1990]). 
midioses, de pastores que conducen a su rebaño por los recónditos lares de la verdad, de lo oculto, de las esencias y del logos. Pero si el proyecto de la 'filosofía post-veritativa' se hace efectivo, se acabarán las filosofías grandilocuentes y sus ínfulas de maestras que no dejan de dar lecciones a los demás. Pues en realidad, piensa Rorty, 'filosofía' no es sino un simple término para designar un tipo de discurso, solo eso. Un discurso cuyas firmas no son Cervantes, Shakespeare, Emerson o Tolstoi, sino Platón, Descartes, Kant o Russell, junto con sus supuestas cuestiones inevitables.

Cuando Rorty se refiere al fin de la filosofía, parece que en realidad apunta a este tipo de problemas de 'manual'. He de decir que Rorty no siempre me parece claro o que, al menos, ha entrecruzado tesis contrapuestas a través de sus diversos escritos. No es fácil obtener un buen condimento con todos los ingredientes filosóficos que menciona, ni siquiera lo es para él. No obstante, hay algo que sí se entiende en lo que defiende y ahí podemos situar su enfoque, a saber: que todas esas cuestiones de manual de 'filosofía veritativa' deben ser evitadas o abandonadas. Retiene el espacio de la literatura para la 'filosofía post-veritativa', mientras que la 'filosofía veritativa', trasnochada, quemada, repleta de cuestiones pueriles (véase Nagel, 1986) tales como la objetividad y la representación, hay que darla por superada. Nada más acerca de la representación, nada sobre la objetividad. La 'filosofía post-veritativa', la prima-hermana de la literatura, ha modificado el vocabulario de los filósofos, quienes ya prefieren hablar en términos de una solidaridad protegida por la hermenéutica secular.

Pero, ¿qué es esto de la posverdad que subyace a la filosofía postveritativa? Como tal, el fenómeno 'posverdad' llama la atención del gran público en 2016, cuando en noviembre el Oxford English Dictionary (OED) elige 'posverdad' (post-truth) como término de ese año. El término captaba bien el espíritu socio-semántico del momento: se enturbia el sentido de los hechos, se abandonan los estándares probatorios o 'evidenciales' en el razonamiento y la argumentación, se miente abiertamente en política y en la academia, etc. (McIntyre, 2018: 1). Si recurrimos al $D R A E$, 'posverdad' es la "distorsión deliberada de una realidad, que manipula creencias y emociones con el fin de influir en la opinión públi-

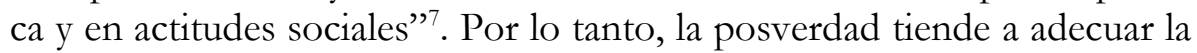
realidad a las propias opiniones de alguien, y no a la inversa (McIntyre, 2018: 5). No se trata tanto de afirmar que los hechos no importan, sino más bien que se pueden moldear, seleccionar y presentar en un contexto

'Pos' no indica la superación de la verdad en el tiempo (como en 'posguerra'), sino el eclipse de la verdad. Esto es, enfatiza su irrelevancia. 
(filosófico, cognoscitivo, político) que favorezca una interpretación de la verdad sobre otras (McIntyre, 2018: 6).

Para entender mejor esto, partamos de una definición básica de 'verdad', la de Aristóteles: "decir de lo que es que no es, o de lo que no es que es, es falso, mientras que decir de lo que es que es, y de lo que no es que no es, es verdadero" (Metafísica, 993b). Esta definición representaría la teoría de la verdad que Rorty encuentra en la 'filosofía veritativa': la teoría de la verdad como 'correspondencia'. Entre las diversas formas de procurar subvertir esta concepción de la verdad, podemos encontrar algunos factores clave de la 'filosofía post-veritativa' de naturaleza literaria o ficcional:

[1] Falsedad no mendaz: si cometemos un error y decimos cosas no verdaderas sin percatarnos de ello, manifestamos una 'falsedad', no una mentira, dado que no hay intención (Frankfurt, 1986; Bengoetxea, 2017).

[2] Ignorancia a voluntad: surge cuando no sabemos realmente si algo es o no es verdadero, pero aun así lo afirmamos sin tomarnos el tiempo y el esfuerzo requeridos para ver si nuestra información es correcta o no (McIntyre, 2018: 7). Aquí se puede culpar al hablante por su pereza, pues si los hechos están disponibles, quien dice algo falso es responsable de su propia ignorancia.

[3] Mentira: se da este caso cuando se afirma una falsedad con la intención de engañar. Toda mentira tiene una audiencia. Cuando la intención del hablante es manipular al oyente para que crea algo que el hablante sabe que es falso, se pasa de la mera interpretación de los hechos a su falsificación.

[4] Indiferencia hacia el valor de verdad: cuando alguien comete bullshit, no necesariamente miente, sino que puede estar mostrando indiferencia hacia el valor de verdad de una afirmación (Frankfurt, 1986; véase Bengoetxea, 2017).

[5] Cinismo: cuando alguien retuerce la verdad para su propia ventaja, sabiendo junto con casi todos los demás que es esto lo que está haciendo realmente, no comete bullshit, pues su intento por influir en los demás es abiertamente deliberado. Es simplemente un cínico (Haack, 2007).

[6] Auto-engaño: es otra forma de posverdad que se da cuando uno se engaña a si mismo hasta creerse una no-verdad que toda fuente creíble pondría en entredicho. En su forma más pura, la posverdad se da cuando uno piensa que la reacción de la audiencia cambia realmente los hechos acerca de una mentira. 
Tanto el 'falseador', el perezoso, el mentiroso, el indiferente y el cínico como el que se auto-engaña y hace 'ver' espejismos al oyente, todos adoptan una forma de posverdad, dado que todas estas son categorías suficientemente hostiles a la verdad (véase Block, 2019: 30-34). Todas son formas post-veritativas. La clave aquí, sin embargo, no es explicar estas formas post-veritativas y ficcionales, pues hemos vivido con ellas desde siempre. Lo nuevo de la era de la posverdad es el desafío no solo a la noción de conocer la realidad, sino a la de aceptar que la hay. El problema radica en la idea de que, en función de lo que alguien quiera que sea verdad, unos hechos importen arbitrariamente más que otros (McIntyre, 2018: 10). Los hechos estarían subordinados a nuestro punto de vista cognoscitivo, político y económico. El conocimiento funcionaría de tal modo que podríamos dudar gratuitamente de un hecho obvio o fácilmente confirmable si obtuviésemos alguna ventaja con ello. Si nuestras creencias se viesen amenazadas por un 'hecho inconveniente', podríamos desafiar el hecho. Esta relación posveritativa con los hechos se da solo cuando buscamos aseverar algo que para nosotros es más importante que la verdad. La ideología se impondría a la verdad al evitar que creamos (o no) en algo en función de las buenas evidencias a favor o en contra. Obviamente, es un enfoque que, aunque consiga una mayor masa crítica -social, académica, política - debido a la facilidad y a la demagogia de sus propuestas y escritos, choca con el sentido común (Haack, 2007). El uso selectivo de los hechos que apuntalan una opinión o posición y el rechazo total de los hechos que no lo hacen parecen herramientas claras para crear una nueva realidad post-veritativa. Los hechos acaban siendo subordinados a la opinión (episteme supeditada a doxa).

Pues bien, muchas de las tácticas empleadas en el mundo postveritativo actual se han recogido de campañas previas, a partir de la década de 1950, de los negadores de la verdad que se oponían al consenso científico ${ }^{8}$. De este modo, al modo de una pendiente realmente resbaladiza, si por ejemplo podemos negar los hechos del cambio climático, esos que Fuller (2018) pone en duda, ¿por qué no dudar de otros hechos? Si la relación entre fumar cigarrillos y desarrollar un cáncer se ha enturbiado durante décadas de desinformación y dudas, ¿por qué no hemos de esperar que lo mismo ocurra con cualquier asunto epistemológico, el cual, diría la filosofía post-veritativa, solo radica en obtener partidarios (es decir, se basa en intereses económicos o políticos)? Esta filosofía ha ampliado su diana, que ahora es la propia realidad. Ya no se conforma con los props de los mundos ficcionales, sino que busca extender la

$8 \quad$ Véase Bengoetxea (2017) para el caso de las campañas de negación de los efectos nocivos del tabaco. 
ficción ontológica a la realidad tout court. Con ello, los factores postveritativos mencionados han conducido, entre otras cosas, a la negación de la ciencia - cambio climático, vacunas, evolución y desarrollo- y parecen haber dado pie al nacimiento de nuevas tácticas para desacreditar toda la realidad fáctica.

Frente a ello, a continuación trataremos de mostrar cómo algunos enfoques cognoscitivos basados en evidencias sí funcionan respecto a la obtención de muchos resultados de conocimiento y en lo referente a aplicaciones prácticas reales para la vida de los seres humanos, enfoques que, en trayectorias probabilistas de aproximación a la objetividad, buscan alejarse de bandos que solo defienden sus intereses y que afirman que todo es relato, ficción articulada en lenguajes, y que la verdad es el resultado de los victoriosos en la lucha entre relatos.

\section{ENSAYOS CLÍNICOS ALEATORIOS, REGULACIÓN Y REALIDAD}

El fenómeno de la posverdad se ha ido conformando durante las últimas décadas, sobre todo en las humanidades y en las ciencias sociales? Una de sus formas específicas es la puesta en cuestión de los procedimientos de evaluación de resultados científicos, en especial la revisión por pares en el caso de las publicaciones. Las reglas de este tipo de revisión son transparentes y responden a un valor cognoscitivo según el cual la evidencia empírica es la cumbre de la evaluación de lo meritorio de una teoría científica (Worrall, 2010). La falibilidad de la ciencia, no obstante, deja lugar a la opción de cometer errores. Por ello, las equivocaciones son tomadas en serio a la hora de destapar fuentes potenciales de sesgo - conflictos de intereses, favorecimiento de intereses de los financiadores, etc.-. Pero esto no ha conseguido que, en el caso en que las creencias ideológicas chocan con las conclusiones de la ciencia, la 'filosofía post-veritativa' (véase Lynch, 2012) deje de responder que los científicos son laxos, interesados o que tan solo se dedican a contarnos 'su' propio relato de cómo es el mundo, hasta el punto de cuestionar tanto los motivos como la competencia de los científicos. De aquí parte el 'negacionismo de la ciencia' (McIntyre, 2018: 18).

\footnotetext{
9 Desgraciadamente, desde el año 2017 algunos autores han detectado más casos de impostura intelectual de corte 'post-veritativo' vinculados a revistas de supuesto prestigio. Son casos que siguen la estela de las denuncias de Alan Sokal en la década de 1990. Dos ejemplos llamativos son los artículos 'Our Struggle is My Struggle: Solidarity Feminism as an Intersectional Reply to Neoliberal and Choice Feminism' (2018), en la revista Afilia: Journal of Women and Social Work, y 'The Conceptual Penis as a Social Construct' (2017), en Cogent Social Sciences.
} 
Es común criticar los resultados científicos por sesgados. Esto da la impresión de que el crítico exige altos estándares científicos y probatorios, pero pocas veces es así. La meta más bien es un intento cínico de socavar la idea de que la ciencia es imparcial y de originar dudas sobre la tesis de que la investigación empírica puede ser realmente 'objetivista' respecto de los valores de los investigadores (Oreskes \& Conway, 2010). Y más aguda si cabe es la crítica de que muchos científicos no manejan buenos estándares científicos, basada en el prejuicio ideológico 'postveritativo' de que son, al igual que sucede en política, simples partidarios del bando que defiende los intereses que solo les sirven a ellos. Según este tipo de objeción, los científicos tan solo nos contarían su propio relato. Sin embargo, parte de esto se basa en una simple confusión o ignorancia (o simplemente bullshit) sobre cómo opera la ciencia. Según esto, es suficiente recopilar evidencias para validar una teoría, de modo que lo que hay que exigir a la ciencia son certidumbres. Sin embargo, la ciencia del siglo XX ha trabajado y trabaja sobre bases probabilistas y de incertidumbre. Más si cabe: la ciencia reguladora debe tomar decisiones o ayudar a tomarlas en condiciones de conocimiento y tiempo limitados (principio de precaución) $^{10}$. Esto nos obliga a conceptualizar la ciencia como una actividad en la que, aunque tengamos muy buenas evidencias, nunca podremos determinar la certeza de una teoría científica. Dada la manera que tenemos de recopilar evidencias científicas (Buchstaller \& Khattab, 2013), siempre será teóricamente posible que alguna parte futura de datos pueda entrar en juego y refutar una teoría.

Esto no significa que las teorías científicas estén injustificadas, que sean una simple trama metafórica o que no merezcan ser creídas. Más bien señala que en algún punto los científicos deberán admitir que incluso sus explicaciones más sólidas no siempre constituyen una verdad definitiva, sino solo creencias gradualmente garantizadas basadas en justificaciones según las evidencias disponibles. Y, de hecho, podemos concebir esta situación epistemológica como una virtud propia de la búsqueda de la verdad. Decir que una teoría científica está bien garantizada, dadas las evidencias disponibles, no es algo trivial ni baladí. Si pretendemos trabajar con los estándares más elevados de explicación empírica, la carga de la prueba (proof) deberá trasladarse a las teorías pseudo-científicas o anti-científicas o post-veritativas que critican a las científicas. Aquí es

10 Klinke, Dreyer, Renn, Stirling \& van Zwanenberg (2006: 375) especifican que hablar de precaución significa que las acciones regulatorias se pueden adoptar en situaciones en las que hay agentes potencialmente perjudiciales que pueden inducir daños a los humanos y al ambiente, pero en las que las pruebas empíricas concluyentes sobre los efectos dañinos no están disponibles en su totalidad. 
donde normalmente entran en juego las evidencias y cuando pedimos al pensador de la 'posverdad' que nos muestre las suyas. Ante este examen, el post-veritativo normalmente se enoja y dice negarse a entrar en lo que simplemente es 'otro juego' de lenguaje, otra forma de relatar (McIntyre, 2018: 20).

Esto es algo que se observa claramente en el caso del cambio climático (Bengoetxea, 2009), campo de cultivo de algo que se viene pergeñando desde la década de 1950 y las controversias 'creadas' en torno al tabaco (Oreskes \& Conway, 2010; Bengoetxea, 2017). La clave primera de estas controversias es clara: los hechos no importan, las evidencias no marcan la diferencia; todo es articulación ficcional, relato. La clave segunda es la indiferencia (Frankfurt, 1986: 39): el valor de verdad de lo dicho es indiferente para quien lo dice porque lo importante es en última instancia generar confusión en el público. Por último, la tercera clave es proteger y ampliar socialmente el mensaje confuso (obtener masa crítica), tal y como hacen muchos políticos e incluso pensadores provocadores cuyas buenas intenciones parecen asfaltar nuestro camino hacia el infierno, como es el caso de Fuller (2018: 22). Un ámbito en el que esta tendencia 'post-veritativa' resulta contrarrestada por las prácticas científicas es el de la medicina.

\subsection{RCTs y medicina}

Hay toda una orientación en filosofía que podríamos denominar 'análisis de los métodos científicos de obtención y generación de evidencias en las ciencias orientadas a la toma de decisiones (ciencias reguladoras)'. Este análisis tiene como trasfondo la asunción de que los ensayos clínicos (o controlados) aleatorios (denominados 'RCT' por sus siglas en inglés; aquí también lo haremos asî) constituyen el mejor medio, a veces el único, para obtener evidencias objetivas e importantes para tomar decisiones en campos como, por ejemplo, la medicina, la farmacología y la alimentación. Con ello se quiere decir que recurrir a las evidencias obtenidas mediante RCTs es una estrategia eficaz que ayuda a desarrollar un conocimiento más objetivo y fiable.

Lo importante, por ende, es que la prueba empírica (evidence) se ha convertido en un concepto fundamental entre los filósofos interesados en explicar cómo integramos los datos en marcos conceptuales y cómo gracias a ellos originamos resultados que son útiles para suministrar mapas del mundo empírico (Achinstein, 2001). Es más, estos datos nos podrían servir también de inputs para tomar decisiones en ámbitos diversos de investigación y de política científica (Achinstein, 2001; Cartwright, 2015). Una forma relevante de obtener evidencias objetivas, no sesgadas 
o garantizadas es precisamente por medio de los mencionados RCTs, los cuales en realidad constituyen la norma de oro probatoria de la medicina científica (Worrall, 2010). La objetividad generada con los RCTs se basa en el uso de grupos de control y de grupos experimentales. Se acepta que los ensayos con medicamentos sobre la base del diseño de RCTs son capaces de mostrar la eficacia del compuesto o medicamento examinado (Rubin, 1974: 689-690; Papineau, 1994). Este es un buen punto de partida para caracterizar una noción de evidencia pertinente para las ciencias reguladoras y que proyecta un procedimiento objetivo no reducible a ideas expresadas en términos de juegos de lenguaje, de idealizaciones alejadas de la 'realidad' o de relatos literarios.

Más allá del alcance fáctico que la aplicación de los RCTs pueda tener a la hora de generar datos, se trata de una aplicación que por lo general funciona y es correcta. Es decir, a modo de regla básica o protocolar, en medicina los RCTs constituyen la mejor herramienta para obtener evidencias científicas objetivas. Estos RCTs son procedimientos en los que se administran tratamientos alternativos para una determinada enfermedad al menos a dos grupos de pacientes con el fin de observar qué tratamiento es el más seguro y el más eficaz. De este modo, los RCTs pueden servir de fundamento para las políticas científicas de base probatoria a la hora de gestionar y tomar decisiones acerca de fármacos. Filosóficamente, los RCTs pueden dar cuerpo a dos nociones epistemológicas clave como son la garantía y la fiabilidad. El regulador tomará una decisión sobre la comercialización de un nuevo medicamento en función de las evidencias que los RCTs proporcionen respecto de la seguridad y eficacia de dicho medicamento (Teira \& Reiss, 2013: 209) ${ }^{11}$. El uso de las mejores evidencias clínicas externas disponibles persigue suministrar más exactitud y precisión en los tests o comprobaciones de diagnósticos, para lo cual mejoran la capacidad de los marcadores prognósticos, así como la eficacia y la seguridad de las dietas terapéuticas y preventivas. Una asunción fundamental que a menudo subyace a este tipo de procedimiento es que los RCTs proporcionan un tipo de objetividad automática, algo que, por ejemplo, el juicio experto individual del médico no podría garantizar por sí mismo.

11 No niego que la práctica de la medicina basada en evidencias integre de hecho la experticia clínica individual del médico (y del enfermero en ciertas ocasiones) con las mejores evidencias disponibles, dado que esto, amén de un desiderátum, también es una práctica cada vez más extendida (coincido en ello con Sackett et al., 1996: 71). No obstante, aquí solo me interesa la parte más 'objetivista' del caso: la investigación clínicamente relevante basada en la ciencia médica o, si se prefiere denominarla más laxamente, en el conocimiento clínico. 


\subsection{Fundamentos 'objetivistas' de los RCTs}

En filosofía de la ciencia se ha asentado un interesante debate acerca de los fundamentos epistémicos de los RCTs. Si este tipo de procedimiento de obtención de evidencias se concibe como lo hace Cartwright (2010), a modo de herramientas para generar inferencias causales, su imparcialidad se puede entender como un rasgo especial e importante para la toma de decisiones reguladoras. Si pudiésemos establecer objetivamente que una causa (un medicamento, por ejemplo) es eficaz para obtener un efecto (curar una enfermedad), el establecimiento del efecto se puede considerar como independiente de cualquier interés que pudiera estar involucrado en un RCT (Cartwright \& Hardie, 2012; Van Baalen \& Boon, 2015). Cartwright considera que los RCTs son básicamente un método garantista para generar declaraciones causales. Con este método, validamos los enunciados causales por medio de un procedimiento deductivo, en el sentido de que, si nuestras asunciones son satisfechas y la evidencia observable es positiva, entonces el enunciado causal se puede considerar verificado.

Supongamos que obtenemos un nuevo tratamiento $(\mathrm{T})$ con el que tratamos una enfermedad previamente intratable e imaginemos que la probabilidad de recuperación $(\mathrm{R})$ en la comunidad de pacientes es mayor entre aquellos que han recibido $T$ que entre los que no lo han hecho (Papineau, 1994: 438): Prob $(\mathrm{R} / \mathrm{T})>\operatorname{Prob}(\mathrm{R} / \neg \mathrm{T})$. Esta correlación nos hace pensar que $T$ causa $R$. En este punto, no obstante, cabe una duda: ¿qué sucedería si la correlación no fuera verdadera porque quienes reciben $\mathrm{T}$ estuvieran, por ejemplo, mejor alimentados (sesgo) que los que no reciben $\mathrm{T}$ ?

Para evitar esta posibilidad de debilitamiento de la generación probatoria, podemos tomar en consideración separadamente a gente mejor y peor alimentada, $\mathrm{y}$ ver si $\mathrm{R}$ sigue estando correlacionada con $\mathrm{T}$ dentro de cada grupo o no: ¿es Prob (R/T \& Y) > Prob $(\mathrm{R} / \neg \mathrm{T} \& \mathrm{Y})$ ? Y ¿es Prob $(\mathrm{R} / \mathrm{T} \& \neg \mathrm{Y})>\operatorname{Prob}(\mathrm{R} / \neg \mathrm{T} \& \neg \mathrm{Y})$ ? Si T no distingue probabilistamente $\mathrm{R}$ entre los individuos mejor alimentados o entre los peor alimentados respectivamente, entonces $\mathrm{T}$ no causa $\mathrm{R}$ (a saber, la correlación era falsa) (Papineau, 1994: 439). Sin embargo, si la desigualdad se mantiene, esto no implica automáticamente que $\mathrm{T}$ causa $\mathrm{R}$, pues puede existir algún factor o variable extraña (confounding) (Abbuhl, Gass \& Mackey, 2013: 117) o alguna otra causa que sea la responsable de la correlación inicial. La cantidad de factores extraños que originen esa correlación puede ser alta y su resolución práctica imposible. Pues bien, aquí es donde se da por sentado que un experimento aleatorio resuelve el problema. Tomamos una muestra de gente con una enfermedad, la dividimos aleatoria- 
mente en dos grupos, de los cuales a uno se le suministra $\mathrm{T}$ y al otro no, y después juzgamos si la probabilidad de recuperación del grupo administrado es mayor que la del otro grupo. Si esto sucede, entonces diremos que $\mathrm{T}$ causa $\mathrm{R}$. La aleatoriedad es lo que nos permite minimizar los factores extraños que pudieran ser responsables de una correlación falsa.

En términos epistemológica y metodológicamente más precisos, decimos que los procedimientos RCT son de carácter deductivo. Para mostrarlo, tan solo requerimos algunas asunciones adicionales que ya Nancy Cartwright ha elaborado a lo largo de sus estudios en filosofía de la ciencia. Veamos:

[A1] Dada la Teoría Probabilista de la Causalidad (TPC) (Suppes, 1970) y algunas variables de naturaleza dicotómica (Cartwright \& Hardie, 2012: 19, 34s; Cartwright, 2010: 60), proponemos la siguiente ecuación de 'fijación causal': para cualquier suceso de tipo-C temporalmente previo a un suceso de tipo-E, "C causa $\mathrm{E}$ si y solo si $\operatorname{Prob}\left(\mathrm{E} / \mathrm{C} \wedge \mathrm{K}_{\mathrm{i}}\right)>$ $\operatorname{Prob}\left(\mathrm{E} / \neg \mathrm{C} \wedge \mathrm{K}_{\mathrm{i}}\right) "$

[A2] Ahora asumimos que los individuos de la muestra están todos regidos por la misma estructura causal descrita mediante una distribución de probabilidad (Prob). Según Cartwright, Prob se define en un espacio de sucesos $\left\{\mathrm{O}, \mathrm{T}, \mathrm{K}_{1}, \mathrm{~K}_{2}, \ldots, \mathrm{K}_{\mathrm{n}}\right\}$ en el que cada $\mathrm{K}_{\mathrm{i}}$ es una descripción de estado del resto de las causas de $\mathrm{O}$, salvo T. En función de estos factores extraños potenciales, podemos atribuir la diferencia restante entre $\operatorname{Prob}\left(\mathrm{O} / \mathrm{T} \wedge \mathrm{K}_{\mathrm{i}}\right)$ y $\operatorname{Prob}\left(\mathrm{O} / \neg \mathrm{T} \wedge \mathrm{K}_{\mathrm{i}}\right)$ al vínculo causal entre $\mathrm{T}$ y $\mathrm{O}$ (Cartwright \& Hardie, 2012: 23; Cartwright, 2010: 61).

Cualquier diferencia en el resultado entre grupos se puede atribuir causalmente a $\mathrm{T}$ en al menos un $\mathrm{K}_{\mathrm{i}}$ relativo a la estructura causal descrita por Prob. Esta conclusión afirma que un RCT ideal se puede hacer encajar deductivamente. Ahora bien, si pretendemos generalizar esta conclusión (validación externa) desde una población experimental $\mathrm{P}_{\mathrm{E}}$ a una población diana general $\mathrm{P}_{\mathrm{G}}$, tendremos que recurrir a asunciones adicionales. $\mathrm{Si}$ queremos establecer que $\mathrm{C}$ causa $\mathrm{E}$ en al menos algunos miembros de $\mathrm{P}_{\mathrm{G}}$, hemos de asumir lo siguiente:

[A3] $\mathrm{C}$ causa $E$ en $\mathrm{K}_{\mathrm{i}}$ syss $\operatorname{Prob}\left(\mathrm{E} / \mathrm{C} \wedge \mathrm{K}_{\mathrm{i}}\right)>\operatorname{Prob}\left(\mathrm{E} / \neg \mathrm{C} \wedge \mathrm{K}_{\mathrm{i}}\right)$, y para cualquier población $\mathrm{A}, \mathrm{C}$ causa $\mathrm{E}$ en $\mathrm{A}$ si y solo si $\mathrm{C}$ causa $\mathrm{E}$ en alguna $\mathrm{K}_{\mathrm{i}}$ que sea un subconjunto de A (Cartwright, 2010: 62). Es decir, al menos una de las subpoblaciones en la que $C$ causa $E$ en $P_{E}$ es una subpoblación de $\mathrm{P}_{\mathrm{G}}$.

Pero puede suceder que dos poblaciones satisfagan una descripción específica $K_{i}$ y al mismo tiempo no estén regidas por los mismos principios causales. Cuando transferimos un principio causal desde una pobla- 
ción que satisface una descripción $\mathrm{K}_{\mathrm{i}}$ a otra que satisface exactamente la misma descripción, hemos de estar seguros de que ambas poblaciones comparten la misma estructura causal (Cartwright, 2010: 66s). Esto exige una asunción más:

[A4] La estructura causal y la medida de probabilidad son las mismas en $\mathrm{P}_{\mathrm{E}} \mathrm{y}$ en $\mathrm{P}_{\mathrm{G}}$.

Coincido con Teira \& Reiss (2013) en que la meta de un RCT no es obtener conclusiones acerca de la causalidad, sino más bien eliminar elementos subjetivos de la experimentación. La aplicación de los RCTs, y la correspondiente disminución del sesgo que ello conlleva es, por lo tanto, una forma adecuada de emplear las evidencias científicas en la medicina 'clínica' con el fin de poder tomar decisiones reguladoras posteriores acerca de muchos tratamientos y suministros de fármacos.

Desde la perspectiva del debate en torno al carácter literario de la filosofía, especialmente de la epistemología, considero que el caso de la medicina, entre otros, sirve para mostrar que las nociones epistemológicas de objetividad, imparcialidad, tendencia a la eliminación del sesgo, tendencia a la eliminación de la subjetividad, etc., en el caso del conocimiento científico, no son nociones baladíes y relativas a marcos o juegos lingüísticos siempre teñidos de convencionalidad y dominio del interés propio, y alejados de las nociones de representación correcta y de verdad. La aleatoriedad, como he dicho previamente, garantiza un alto grado de imparcialidad o minimización del sesgo en los ensayos médicos para determinar los efectos de ciertos tratamientos y fármacos. Si concebimos los RCTs como herramientas reguladoras, podemos llegar a la conclusión de que son bastante exitosas en la práctica real de las ciencias orientadas a la toma de decisiones, basada en el conocimiento causal de fondo de los ensayos clínicos, así como en el control de las lagunas posibles que pudieran surgir en torno a la validez externa.

\section{CODA FINAL}

Desde una perspectiva del argumento correcto y, o, de los argumentos erigidos sobre evidencias, el programa de Rorty y las propuestas de la posverdad no solo son falaces, sino que aparentan constituir una controversia 'agnotológica' (Proctor, 1995) estéril propia de los creadores de tantas iniciales de apariencia importante (STS (science and technology studies), SSK (social studies of knowledge), NLF (new literature forms)). Estas parecen erigirse sobre argumentos correctos, pero hay errores ocultos más o menos deliberadamente. Defender que las investigaciones filosóficas son en realidad propuestas narrativas y que los conceptos de verdad y de representación son solo fábulas nos obligaría a admitir que la literatura cientí- 
fica y filosófica es literatura en sentido literal y expresiones hipónimas de 'narrativa' (Haack, 2007: 208 y sigs.). Es francamente difícil dar sentido a esto.

Los intentos de convertir la filosofía en conocimiento literario y de ficción, al menos de forma rigurosa, chocan de frente con la visión del mundo que tienen las disciplinas científicas y el sentido común. De hecho, Rorty y los defensores de la posverdad olvidan que el conocimiento humano es un hecho natural que se constituye y produce junto con otros hechos naturales. El 'conversacionalismo' (todo es conversación) y el 'textualismo' (todo es texto) han olvidado de alguna manera la biología, ciencia ante la cual no hemos encontrado nada que responda mejor a nuestros deseos epistemológicos. Han olvidado, por ende, que la razón y el lenguaje son productos de la evolución natural ${ }^{12}$, que el conocimiento no es solo un proceso de carácter lingüístico que únicamente persigue representar algo fuera del propio lenguaje o de la mente. Pero precisamente hay que mirar al otro lado de la moneda: que el conocimiento también depende y se constituye gracias a las acciones realizadas en el entorno y a las manipulaciones desarrolladas en este, pues el conocimiento es una forma de adecuarse al entorno, tal vez la mejor capacidad para ello del ser humano. Y dicho entorno se extiende más allá del lenguaje y de los vocabularios. Como tal, el discurso científico, pero también el filosófico (sin que la filosofía se tenga que reducir a ciencia), son dependientes de ciertas constricciones naturales. Algunas de ellas son las propias evidencias con las que nos topamos en el mundo. Su definición, poder verlas con más nitidez, no equivale a construirlas ex nibilo, al modo idealista que, con pretensiones de notoriedad y novedad, la posverdad y Rorty, entre otros, defienden. Las operaciones de los discursos científico y filosófico están vinculadas irremediablemente a dichas constricciones, siempre bajo la mirada atenta del pensamiento crítico, argumentado. El discurso literario, en cambio, no tiene por qué estar supeditado a las mencionadas constricciones. La ficción es otra cosa (véase Stock, 2017). La actividad del discurso filosófico, al igual que la vida real y no psicológicamente patológica de los individuos, está condicionada por el entorno, el cual no es sino la Naturaleza, la realidad, el mundo, las leyes lógicas, por ingenuos y anticuados que estos términos aparenten ser para la posverdad.

Los seres humanos hemos demostrado que sí hay algo que merece la pena investigar más allá del lenguaje y de la conversación, hagámoslo de forma sofisticada y sistemática, teórica y empírica, científica y filosófica,

12 Es decir, la naturaleza se puede situar por delante del lenguaje y del pensamiento (véase Bickerton, 1990). A esta transformación se la denomina 'naturalización'. 
o mediante el sentido común. Las formas más sistemáticas y propiamente científico-filosóficas requieren un theorein estricto, o eso persiguen; la forma más popular del sentido común solo exige un theorein laxo. El conocimiento de sentido común nos sirve ante todo para la vida cotidiana; toma la forma del conocimiento popular (folk knowledge): psicología popular, física popular, etc. Tanto este tipo de conocimiento como el más sofisticado son 'objetivistas' en la medida en que tienden a encontrar algo más allá del lenguaje, de la mente o del sujeto. Actúan de forma pragmática y presuponen obviamente la existencia de ese algo. Y esto funciona. Pues bien, los defensores de la posverdad olvidan este presupuesto y los resultados prácticos que gracias a él se obtienen y que, si somos fieles al lema de sus antecesores los pragmatistas norteamericanos, la verdad tiene que ver con el éxito como meta a plazo futuro que se constata una y otra vez. En definitiva, el conocimiento está repleto de acciones lingüísticas, pero también de acciones no-lingüísticas.

Hacer ciencia no solo es crear relatos e inscripciones (Latour, 2001). Algunos consideran que la filosofía sí podría consistir en algo similar al menos. Pero no logro ver cómo los filósofos pueden llegar a escribir mejores libros o relatos que los poetas o los 'literatos' dedicados a ello. La filosofía, no nos engañemos, no es un género literario, como tampoco lo era la 'escritura' o la impresión de los antiguos listines telefónicos. Decir lo contrario sería faltar al respeto a la propia literatura. Ahora bien, esta tampoco es investigación. Reducir la realidad a ficción o viceversa no tiene sentido. Sabemos que la metáfora y la fábula, y otras figuras literarias, son parasitarias de la realidad, pues comprendemos aquellas gracias a esta en su mayor parte, y no a la inversa - la metáfora requiere un trasfondo de verdad y de verosimilitud para poder ser comprendida (Currie, 1990)—. No habría ficción sin verdad ni realidad. Y aceptamos que sí hay ficciones ${ }^{13}$.

Todo esto permite que identifiquemos algunas dudas, también profundas, respecto del deseo 'post-veritativo' rortyano de deshacernos de la epistemología. Mencionaré tres:

Por un lado, la 'filosofía post-veritativa' tampoco parece librarse de las dicotomías de la 'filosofía veritativa' que a Rorty tanto molestan. Rorty solo sustituye las antiguas dicotomías con otras menos glamurosas: objetividad versus solidaridad, metafísica versus consenso, verdad versus relato. Tan solo parecen ser los ingredientes de un montaje retórico indisimulado. De floritura lingüística abundante, recuerda al discurso de pasatiempo de la hora de tomar el té, como apuntaba Russell acerca de las

13 El tratamiento filosófico de la ficción 'cognoscitiva' merece un capítulo aparte que aquí no podemos desarrollar. Véase, no obstante, Toon (2012). 
Investigaciones filosóficas ${ }^{14}$. Es más, en la filosofía de la mente actual, así como en las ciencias cognitivas, la dicotomía tan denostada 'mente-cerebro' ha seguido abriendo nuevas vías de indagación, como son los enfoques de los sistemas complejos y los 'mecanísticos' (Dennett, 1986), los cuales pretenden dejar atrás, entre otras cosas, las concepciones metafóricas y de corte literario.

Además, ¿qué envejece un vocabulario? Si hemos de rechazar la dicotomía 'objetivo-subjetivo', no lo envejece nada objetivo. En el ideario de Rorty, el valor del vocabulario es práctico y, de hecho, observa que los vocabularios filosóficos ya no pesan apenas en la cultura y sociedad de hoy. No sucede lo mismo con los vocabularios literarios. Pero, ¿podemos seguir pretendiendo ser cultos si dejamos de lado el conocimiento filosófico acerca de la ciencia, sobre todo tras la obra de Snow? En Las dos culturas y un nuevo enfoque (1959), Snow incidió en la importancia del conocimiento científico en la cultura del siglo XX, y yo añadiría algo más: el esfuerzo y las capacidades que el conocimiento científico y la filosofía que reflexiona sobre él exigen hace que muchas afirmaciones post-veritativas tan solo sean formas disfrazadas de generar, sin excesiva rigurosidad ni trabajo, eslóganes atractivos o provocadores. Pues ahí está la biología, o las reflexiones en torno a la mente, y las filosofías basadas en estudios de evidencias acerca de la ciencia y de la tecnología. Pero nos piden que de repente las tomemos y las vertamos por el sumidero, lo cual no sería sino algo parecido a realizar una filosofía apriorista sin mucha razón de ser hoy. Afirmar que es el vocabulario literario el más actualizado en el mundo del pensamiento es absurdo y no se adecua a la realidad y a las prácticas cognoscitivas cotidianas en absoluto.

Por último, considero que los conceptos erigidos y manejados por la 'filosofía veritativa' (verdad, representación, realidad, etc.) aún son merecedores de atención y uso, aunque, por supuesto, desde perspectivas novedosas, actualizadas, que tengan en cuenta los avances cognoscitivos y el desarrollo social, ante todo el científico, el cual, en términos rortyanos, sí nos suministraría el vocabulario más avanzado. La verdad y la representación, más allá de las metáforas que pudieran rodearles, han sido objeto de sesudas investigaciones teóricas, básicamente filosóficas, y muchas de ellas de gran interés conceptual. El uso de metáforas internas a esos discursos no es nada extraño en el mundo del conocimiento,

14 En su reseña de tono irónico sobre las Investigaciones filosóficas de Wittgenstein, Russell decía lo siguiente sobre el tipo de filosofía que el austríaco proponía: "a lo sumo, la filosofía es una ayuda mínima para el lexicógrafo; en el peor de los casos, es un pasatiempo estúpido para la hora de tomar el té” (Russell, 1956: 615). 
siempre atento a intentar eliminarlas como se dejan caer los lastres de un globo aerostático en alzada.

\section{REFERENCIAS}

Abbuhl, R., Gass, S., \& Mackey, A. (2013). Experimental research design. En R. Podesva \& D. Sharma (Eds.), Research Methods in Linguistics (pp. 116-134). Cambridge: Cambridge University Press.

Achinstein, P. (2001). The Book of Evidence. Oxford: Oxford University Press.

Anderson, M. (1996). Impostors in the Temple: A Blueprint for Improving Higher Education in America. Washington: Hoover Institution Press.

Baker, E. \& Oreskes, N. (2017). Science as a game, marketplace or both: A reply to Steve Fuller. Social Epistemology Review and Reply Collective, 6(9), 6569.

Ball, J. (2017). Post-Truth: How Bullshit Conquered the World. Londres: Biteback.

Bengoetxea, J. B. (2009). Teknozientzia, politika zientifikoa eta bullshit. Jakin, 171(2), 69-82.

Bengoetxea, J. B. (2017). A Gricean analysis of discoursive strategies in decision-oriented science: Bullshit, uncertainty, and meaning. Filosofia Unisinos, 18(1), 24-35.

Bickerton, D. (1990). Lenguaje y especies. (Miguel A. Valladares, Trad.) Madrid: Alianza.

Block, D. (2019). Post-Truth and Political Discourse. Cham: Palgrave-Macmillan.

Buchstaller, I. \& Khattab, G. (2013). Population samples. En R.J. Podesva \& D. Sharma (Eds.), Research Methods in Linguistics (pp. 74-95). Cambridge: Cambridge University Press.

Carnap, R. (1928). La construcción lógica del mundo. (Laura Mues de Schrenk, Trad.) México: UNAM.

Cartwright, N. (2010). What are randomised controlled trials good for? Philosophical Studies, 147, 59-70.

Cartwright, N. (2015). Evidence: For Policy, and Wheresoever Rigor is a Must. London: LSE.

Cartwright, N. \& Hardie, J. (2012). Evidence-Based Policy: A Practical Guide to Doing It Better. Oxford: Oxford University Press.

Currie, G. (1990). The Nature of Fiction. Cambridge: Cambridge University Press.

Dennett, D. C. (1986). The Logical Geography of Computational Approaches: A View from the East Pole. En M. Brand \& R.M. Harnish (Eds.), The Representation of Knowledge and Belief (pp. 59-79). Tucson: The University of Arizona Press.

Derrida, J. (1987). La desconstrucción en las fronteras de la filosofía. (Patricio Peñalver, Trad.) Barcelona: Paidós.

Frankfurt, H. G. (1986). On Bullshit. Princeton: Princeton University Press.

Fuller, S. (2018). What Can Philosophy Teach Us About the Post-truth Condition. En M.A. Peters, S. Rider, M. Hyvönen \& T. Besley (Eds), Post-Truth, 
Fake News: Viral Modernity \& Higher Education (pp. 13-25). Singapore: Springer Nature.

Haack, S. (2007). Defending Science -Within Reason: Between Scientism and Cynicism (Segunda edición). Amherst, NY: Prometheus Books.

Heidegger, M. (1976). La autoafirmación de la Universidad alemana. (Ramón Rodríguez, Trad.) Madrid: Tecnos.

Horkheimer, M. (1937). Der Neueste Angriff auf die Metaphysik. En M. Horkheimer (1979), Die gesellschaftliche Funktion der Pbilosophie (pp. 90-144). Frankfurt: Surhkamp.

Klinke, A., Dreyer, M., Renn, O., Stirling, A. \& van Zwanenberg, P. (2006). Precautionary Risk Regulation in European Governance. Journal of Risk Research, 9(4), 373-392.

Kusch, M. (1989). Language as Calculus vs. Language as Universal Medium: A Study in Husserl, Heidegger and Gadamer. Dordrecht: Kluwer.

Latour, B. (2001). La esperanza de Pandora (Tomás Fernández, Trad.). Barcelona: Gedisa.

Lyotard, J.-F. (1987). La condición postmoderna (Mariano Antolín, Trad.). Madrid: Cátedra.

Lynch, M. (2012). In Praise of Reason. Cambridge, MA: MIT Press.

Margolis, J. (1991). The Truth about Relativism. Oxford: Basil Blackwell.

McIntyre, L (2018). Post-Truth. Cambridge, Mass.: The MIT Press.

Moya, E. (2002). Filosofía, literatura y verdad. Revista de Filosofía, 27(2), 305-336.

Nagel, T. (1986). The View from Nowhere. New York: Oxford University Press.

Nietzsche, F. (1873). Sobre verdad y mentira en sentido extramoral y otros fragmentos de filosofía del conocimiento (Luis M. Valdés, Teresa Orduña, Trads.). Madrid: Tecnos.

Oreskes, N. \& Conway, E. (2010). Merchants of Doubts: How a Handful of Scientists Obscured the Truth on Issues from Tobacco Smoke to Global Warming. New York: Bloomsbury.

Papineau, D. (1994). The Virtues of Randomization. The British Journal for the Pbilosopby of Science, 45(2), 437-450.

Pardo, J. L. (2002). Más Prozac y menos Platón. Archipiélago, (50), 11-15.

Proctor, R. N. (1995). Cancer wars: How politics shapes what we know and don't know about cancer. New York: Basic Books.

Rorty, R. (1982). Consequences of Pragmatism, (Essays: 1972-1980). Brighton: The Harvester Press Limited.

Rorty, R. (1998). Truth and Progress. Philosophical Papers, Vol. 3. Cambridge: Cambridge University Press.

Rorty, R. (2000). Filosofia y futuro (Angela Ackermann, Trad.). Barcelona: Gedisa. Rubin, D. (1974). Estimating Causal Effects of Treatments in Randomized and Nonrandomized Studies. Journal of Educational Psychology, 66(5), 688-701.

Russell, B. (1956). Philosophical Analysis. En J.G. Slater (Ed.), The Collected Papers of Bertrand Russell, Vol. 13 (pp. 614-625). London: Routledge. 
Sackett, D., Rosenberg, W., Gray, J., Haynes, R., \& Richardson, W. (1996). Evidence based medicine: what it is and what it isn't. British Medical Journal, 312, 71-72.

Stock, K. (2017). Learning from Fiction and Theories of Fictional Content. Teorema, 35(3), 69-85.

Suppes, P. (1970). A Probabilistic Theory of Causality. Amsterdam: North-Holland.

Teira, D., \& Reiss, J. (2013). Causality, Impartiality and Evidence-Based Policy. In H. Chao, S. Chen \& R. Millstein (Eds.), Mechanism and Causality in Biology and Economics (pp. 207-224). Dordrecht: Springer.

Toon, A. (2012). Models as Make-Believe: Imagination, Fiction and Scientific Representation. Basingstoke: Palgrave-Macmillan.

Van Baalen, S., \& Boon, M. (2015). An epistemological shift: from evidencebased medicine to epistemological responsibility. Journal of Evaluation in Clinical Practice, 21, 433-439.

Worrall, J. (2010). Evidence: philosophy of science meets medicine. Journal of Evaluation in Clinical Practice, 16, 356-362. 